\title{
TRANSPORT-EQUILIBRIUM SCHEMES FOR COMPUTING CONTACT DISCONTINUITIES IN TRAFFIC FLOW MODELING*
}

\author{
CHRISTOPHE CHALONS ${ }^{\dagger}$ AND PAOLA GOATIN ${ }^{\ddagger}$
}

\begin{abstract}
We present a very efficient numerical strategy for computing contact discontinuities in traffic flow modeling. We consider the Aw-Rascle model, and the objective is to remove spurious oscillations generated for instance by the Godunov method near contact discontinuities. The method is mixed and based on both a random sampling strategy and the Godunov method. To prove the validity of the method, we show that it enjoys important stability properties and propose numerical tests. The convergence of the algorithm is demonstrated numerically.
\end{abstract}

Key words. hyperbolic conservation laws, continuous traffic models, contact discontinuities, transport-equilibrium scheme

AMS subject classifications. 35L60, 35L65, 65M99

\section{Introduction}

Mathematical and numerical models for traffic dynamics can be developed under different approaches. We can distinguish between microscopic (particle-based), mesoscopic (gas-kinetic) and macroscopic (fluid-dynamic) models. Here, we are interested in continuum models, which are based on conservation (or balance) equations. Firstorder models consist of one equation, describing the conservation of mass (i.e. the number of cars). The prototype of these models is due to Lighthill-Whitham [17] and Richards [20] (LWR). If another conservation equation is added, we obtain a so-called second-order model. A first prototype was proposed by Payne [19] and Whitham [24]. This kind of models mimics the isentropic Euler equations of fluid mechanics, requiring conservation of mass and momentum. However, traffic flow does not behave as usual fluids, and the Payne-Whitham model shows some peculiarites, as pointed out by Daganzo [12]. In order to correct these drawbacks, Aw and Rascle [3] proposed another model, in which they replace the space derivative of the "pressure" in the momentum equation by the convective derivative $\partial_{t}+v \partial_{x}$, where $v$ stands for the (mean) car velocity. More precisely, the model consists of a $2 \times 2$ system of conservation laws for the car density $\rho$ and the "momentum" $y$ which reads as follows:

$$
\left\{\begin{array}{l}
\partial_{t} \rho+\partial_{x}(\rho v)=0, \\
\partial_{t} y+\partial_{x}(y v)=0,
\end{array} \quad x \in \mathbb{R}, t>0 .\right.
$$

The conservative variable $y$ is defined by

$$
y=\rho w, \quad w=v+p(\rho) .
$$

Here $p$ is a "pressure" term (as in gas dynamics) which takes into account drivers' reactions to the state of traffic in front of them. In this way, if we suitably choose the domain in the phase space, the model satisfies the following basic properties: no information travels faster than cars, and density and velocity remain non-negative and bounded.

\footnotetext{
${ }^{*}$ Received: December 21, 2006; accepted (in revised version): April 27, 2007. Communicated by Lorenzo Pareschi.

${ }^{\dagger}$ Université Denis Diderot-Paris 7 \& Université Pierre et Marie Curie-Paris 6, UMR 7598 LJLL, Paris, F-75005, France (chalons@math.jussieu.fr).

${ }^{\ddagger}$ Laboratoire d'Analyse Non linéaire Appliquée et Modélisation, I.S.I.T.V., Université du Sud Toulon - Var, B.P. 56, 83162 La Valette du Var Cedex, France (goatin@univ-tln.fr).
} 
The velocity $v$ is linked to the conservative unknown $\mathbf{u}=(\rho, y)$ by $(1.2)$ and a closure relation for $p$. Following [3], the function $p$ has to be chosen as a smooth and strictly increasing function such that $\rho p(\rho)$ is strictly convex. For instance, we will take (without any restriction) the following definition for the numerical experiments of this paper:

$$
p(\rho)=v_{\text {ref }} \ln \left(\frac{\rho}{R}\right),
$$

where $R$ is the maximal car density allowed by the road, and $v_{r e f}$ is a given reference velocity. Introducing now three parameters $v_{M}$ and $w_{m}, w_{M}$ for the thresholds of $v$ and $w$ respectively, we define the following invariant region for (1.1):

$$
\Omega=\left\{(\rho, y) \in \mathbb{R}^{2} \text { with } \rho \in[0, R], v \in\left[0, v_{M}\right], w \in\left[w_{m}, w_{M}\right]\right\} .
$$

We observe that (1.1) can also be written in condensed form as

$$
\partial_{t} \mathbf{u}+\partial_{x} \mathbf{f}(\mathbf{u})=0, \quad \mathbf{u} \in \Omega,
$$

where the flux function $\mathbf{f}$ finds a clear definition from previous developments.

As detailed in Section 2, the system under consideration is strictly hyperbolic for $\rho>0$, with a genuinely nonlinear and a linearly degenerate characteristic field. The latter is associated with the faster eigenvalue which is equal to $v$ and then develops discontinuous waves, the so-called contact discontinuities, for which the speed of propagation is continuous and given by $v$. In this paper, we will focus on the numerical approximation of these contact discontinuities.

In the past decade, the numerical approximation of contact discontinuities received much attention in the context of compressible multicomponent (or multifluid) flows. Indeed, when the flow is made of several species, it is observed that classical conservative schemes (like Godunov's scheme) generate important nonphysical oscillations near the material fronts, eventually leading to numerical solutions that are not precise (at least for realistic meshes). The same pathologies also exist for single fluid computations, and they appear for system (1.1). Several cures have been proposed in the literature, see for instance $[15,16,1,21,13,2,4]$ and the references therein. Roughly speaking, the common idea is to retain a classical conservative scheme far from the material interfaces and to introduce a non-conservative modification in the regions where the problem occurs, in order to preserve constant pressure and velocity. Note however that the threshold technique often attached to the local treatment prevents the methods from strictly preserving isolated contact discontinuities. The resulting non-conservative schemes give good results and seem to be numerically convergent. Note also that these strategies are usually designed for models involving at least two fluids and two pressure laws. As a consequence, it seems difficult to apply them to our "single fluid" system (1.1). We mention, however, that in [7] the authors will propose a mixed Lagrangian/Eulerian approach that applies also for closure relations involving a single pressure law.

Based on a recent work [5] (see also [6]) by the first author, we present in this paper an algorithm whose objective is to remove the spurious oscillations generated by the Godunov scheme (for instance) near the contact discontinuities seperately of system (1.1). The method proposes to treat contact discontinuities using a random sampling strategy, and to retain the Godunov scheme for the other waves. As expected, the whole algorithm is non-conservative but numerical experiments give very 
good numerical solutions with sharp (without numerical diffusion) contact discontinuities and very small conservation errors, and show numerical convergence. Moreover, we are able to prove that the method enjoys important stability properties like strong consistency and a maximum principle for the two Riemann invariants of system (1.1), see theorems 3.1 and 3.2 in Section 3.3. Note also that our algorithm is free of threshold techniques. As a consequence of all these properties, contact discontinuities are always computed without oscillations.

To conclude this section, we recall that another second-order model has been proposed by Colombo [10]. This model has a genuinely nonlinear and a linearly degenerate field. Thus, the same difficulties related to the numerical contact discontinuities are expected to occur. Moreover, both models [10,3] have been coupled with the LWR equation, giving rise to traffic flow models with phase transitions [11, 14]. The techniques presented in this paper can easily be adapted and used in these cases. In particular, for models with phase transitions, an efficient numerical scheme has been proposed by the authors in [8]. It can be combined with the present scheme in order to better approximate contact discontinuities.

\section{Basic properties of the Aw-Rascle model}

In this section, we briefly recall the basic features of the model under consideration and we refer the reader to [3] (see also [14]) for more details, knowing that the properties here stated follow from usual considerations. The basic information on the Aw-Rascle system is collected in the following table:

$$
\begin{array}{ll}
r_{1}(\rho, v)=\left[\begin{array}{c}
1 \\
-p^{\prime}(\rho)
\end{array}\right], & r_{2}(\rho, v)=\left[\begin{array}{l}
1 \\
0
\end{array}\right], \\
\lambda_{1}(\rho, v)=v-\rho p^{\prime}(\rho), & \lambda_{2}(\rho, v)=v, \\
\nabla \lambda_{1} \cdot r_{1}=-2 p^{\prime}(\rho)-\rho p^{\prime \prime}(\rho), & \nabla \lambda_{2} \cdot r_{2}=0 \\
\mathcal{L}_{1}\left(\rho ; \rho_{o}, v_{o}\right)=v_{o}+p\left(\rho_{o}\right)-p(\rho), & \mathcal{L}_{2}\left(\rho ; \rho_{o}, v_{o}\right)=v_{o} \\
w_{1}=v+p(\rho), & w_{2}=v,
\end{array}
$$

where $r_{i}$ is the $i$-th right eigenvector, $\lambda_{i}$ the corresponding eigenvalue and $\mathcal{L}_{i}$ is the $i$-th Lax curve. We note that shock and rarefaction curves coincide, hence the system belongs to the Temple class [23]. The Riemann invariants associated with each eigenvalue $\lambda_{1}$ and $\lambda_{2}$ are $w_{1}=v+p(\rho)$ and $w_{2}=v$ respectively. In addition, it is easily seen that the first characteristic field is genuinely nonlinear, and the second is linearly degenerate. Therefore, depending on the initial data, the self-similar solution to the general Riemann problem

$$
\left\{\begin{array}{l}
\partial_{t} \mathbf{u}+\partial_{x} \mathbf{f}(\mathbf{u})=0 \\
\mathbf{u}(x, 0)=\left\{\begin{array}{l}
\mathbf{u}^{l} \text { if } x<0, \\
\mathbf{u}^{r} \text { if } x>0
\end{array}\right.
\end{array}\right.
$$

will consist of one Lax wave (shock or rarefaction) moving with negative and/or positive speeds, and a contact discontinuity always moving with positive speed $v$. For a more detailed description of the Riemann solver see again [3]. Using the Riemann coordinates and the property that $w_{1}$ (respectively $w_{2}$ ) is constant across the waves of the first (respectively second) family, the intermediate state $\mathbf{u}^{\star}\left(\mathbf{u}^{l}, \mathbf{u}^{r}\right)$ in the Riemann solution is easily computed:

$$
\left\{\begin{array} { l } 
{ w _ { 1 } ^ { \star } = w _ { 1 } ^ { l } = v ^ { l } + p ( \rho ^ { l } ) , } \\
{ w _ { 2 } ^ { \star } = w _ { 2 } ^ { r } = v ^ { r } , }
\end{array} \Longrightarrow \left\{\begin{array}{l}
\rho^{\star}=\rho^{l} \exp \left(\frac{v^{l}-v^{r}}{v_{r e f}}\right), \\
y^{\star}=\rho^{\star}\left(v^{r}+p\left(\rho^{\star}\right)\right) .
\end{array}\right.\right.
$$


Of course, we note that $v^{\star}:=v\left(\mathbf{u}^{\star}\left(\mathbf{u}^{l}, \mathbf{u}^{r}\right)\right)=v^{r}$. We will use the above cheap computation in our algorithm. In contrast, we will not compute directly rarefaction waves.

It is also important for the forthcoming developments to notice that the solution to (2.2) obeys a maximum principle for the two Riemann invariants. In other words, if $(x, t) \rightarrow \mathbf{u}_{\mathbf{r}}\left(\frac{x}{t} ; \mathbf{u}^{l}, \mathbf{u}^{r}\right)$ denotes the self-similar solution of $(2.2)$, we have

$$
\left\{\begin{array}{l}
\inf \left\{v^{l}+p\left(\rho^{l}\right), v^{r}+p\left(\rho^{r}\right)\right\} \leq(v+p)\left(\mathbf{u}_{\mathbf{r}}\left(\frac{x}{t} ; \mathbf{u}^{l}, \mathbf{u}^{r}\right)\right) \leq \sup \left\{v^{l}+p\left(\rho^{l}\right), v^{r}+p\left(\rho^{r}\right)\right\}, \\
\inf \left\{v^{l}, v^{r}\right\} \leq v\left(\mathbf{u}_{\mathbf{r}}\left(\frac{x}{t} ; \mathbf{u}^{l}, \mathbf{u}^{r}\right)\right) \leq \sup \left\{v^{l}, v^{r}\right\} .
\end{array}\right.
$$

\section{Numerical approximation}

We first briefly recall the Godunov scheme applied to system (1.1), showing that it introduces nonphysical oscillations near contact discontinuities, which can significantly affect the whole approximate solution. To overcome this problem, we will introduce a new method in which we evolve separately the contact discontinuities and the shock or rarefaction waves.

Let $\Delta x$ and $\Delta t$ be two constant increments for space and time discretizations, and let $\nu=\Delta t / \Delta x$. We then define the mesh interfaces $x_{j+1 / 2}=j \Delta x$ and the cell centers $x_{j}=(j+1 / 2) \Delta x$ for $j \in \mathbb{Z}$, the intermediate times $t^{n}=n \Delta t$ for $n \in \mathbb{N}$, and at each time $t^{n}$ we denote $\mathbf{u}_{j}^{n}$ an approximate mean value of the solution of (1.5) on the interval $\mathcal{C}_{j}=\left[x_{j-1 / 2}, x_{j+1 / 2}\right), j \in \mathbb{Z}$. In other words, a piecewise constant approximation $x \rightarrow$ $\mathbf{u}_{\nu}\left(x, t^{n}\right)$ of the solution $\mathbf{u}$ is given by

$$
\mathbf{u}_{\nu}\left(x, t^{n}\right)=\mathbf{u}_{j}^{n} \text { for all } x \in \mathcal{C}_{j}, j \in \mathbb{Z}, n \in \mathbb{N} .
$$

When $n=0$, we set

$$
\mathbf{u}_{j}^{0}=\frac{1}{\Delta x} \int_{x_{j-1 / 2}}^{x_{j+1 / 2}} \mathbf{u}_{0}(x) d x, \text { for all } j \in \mathbb{Z}
$$

where $\mathbf{u}_{0} \in \Omega$ is a given initial data.

Assuming as given a sequence $\left(\mathbf{u}_{j}^{n}\right)_{j \in \mathbb{Z}}$ at time $t^{n}$, we concentrate now on the computation of an approximate solution at the next time level $t^{n+1}$.

3.1. The Godunov scheme. As is well-known, the Godunov scheme writes as follows

$$
\mathbf{u}_{j}^{n+1}=\mathbf{u}_{j}^{n}-\frac{\Delta t}{\Delta x}\left(\mathbf{f}_{j+1 / 2}^{n}-\mathbf{f}_{j-1 / 2}^{n}\right) \text { for all } j \in \mathbb{Z},
$$

where the numerical fluxes are such that

$$
\mathbf{f}_{j+1 / 2}^{n}=\mathbf{f}\left(\mathbf{u}_{\mathbf{r}}\left(0^{-} ; \mathbf{u}_{j}^{n}, \mathbf{u}_{j+1}^{n}\right)\right) \text { for all } j \in \mathbb{Z} .
$$

Let $\mathbf{v}$ be the solution for times $t \in[0, \Delta t]$ of (1.1) with piecewise constant initial data $\mathbf{u}_{0}(x)=\mathbf{u}_{\nu}\left(x, t^{n}\right)$. Under the usual CFL restriction

$$
\frac{\Delta t}{\Delta x} \max _{\mathbf{u}}\left\{\left|\lambda_{i}(\mathbf{u})\right|, i=1,2\right\} \leq \frac{1}{2}
$$

for all the $\mathbf{u}$ under consideration, $\mathbf{v}$ is obtained by gluing together the solutions of the Riemann problems set at each interface. More precisely

$$
\mathbf{v}(x, t)=\mathbf{u}_{\mathbf{r}}\left(\frac{x-x_{j+1 / 2}}{t} ; \mathbf{u}_{j}^{n}, \mathbf{u}_{j+1}^{n}\right) \text { for all }(x, t) \in\left[x_{j}, x_{j+1}\right] \times[0, \Delta t] .
$$


See Fig. 3.1 for an illustration. Green's formula tells us that (3.2) is equivalent to averaging the function $x \rightarrow \mathbf{v}(x, \Delta t)$ on the interval $\left[x_{j-1 / 2}, x_{j+1 / 2}\right]$ :

$$
\mathbf{u}_{j}^{n+1}=\frac{1}{\Delta x} \int_{x_{j-1 / 2}}^{x_{j+1 / 2}} \mathbf{v}(x, \Delta t) d x, j \in \mathbb{Z} .
$$

An equivalent way to recover (3.2) consists in setting

$$
\mathbf{u}_{j}^{n+1}=\frac{1}{2}\left(\mathbf{u}_{j+1 / 2, L}^{n+1}+\mathbf{u}_{j-1 / 2, R}^{n+1}\right),
$$

where

$$
\mathbf{u}_{j+1 / 2, L}^{n+1}=\frac{2}{\Delta x} \int_{x_{j}}^{x_{j+1 / 2}} \mathbf{u}_{\mathbf{r}}\left(\frac{x-x_{j+1 / 2}}{\Delta t} ; \mathbf{u}_{j}^{n}, \mathbf{u}_{j+1}^{n}\right) d x=\mathbf{u}_{j}^{n}-\frac{2 \Delta t}{\Delta x}\left(\mathbf{f}_{j+1 / 2}^{n}-\mathbf{f}\left(\mathbf{u}_{j}^{n}\right)\right)
$$

and

$$
\mathbf{u}_{j+1 / 2, R}^{n+1}=\frac{2}{\Delta x} \int_{x_{j+1 / 2}}^{x_{j+1}} \mathbf{u}_{\mathbf{r}}\left(\frac{x-x_{j+1 / 2}}{\Delta t} ; \mathbf{u}_{j}^{n}, \mathbf{u}_{j+1}^{n}\right) d x=\mathbf{u}_{j+1}^{n}-\frac{2 \Delta t}{\Delta x}\left(\mathbf{f}\left(\mathbf{u}_{j+1}^{n}\right)-\mathbf{f}_{j+1 / 2}^{n}\right)
$$

denote the averages of the Riemann solution $x \rightarrow \mathbf{u}_{\mathbf{r}}\left(\frac{x-x_{j+1 / 2}}{\Delta t} ; \mathbf{u}_{j}^{n}, \mathbf{u}_{j+1}^{n}\right)$ on the halfcells $\left[x_{j}, x_{j+1 / 2}\right]$ and $\left[x_{j+1 / 2}, x_{j+1}\right]$. This point of view will be useful in the following.

3.2. Failure of Godunov scheme in properly capturing contact discontinuities. Let us consider the Riemann problem (2.2) with $\mathbf{u}^{l}=\left(\rho^{l}, y^{l}\right)$ and $\mathbf{u}^{r}=\left(\rho^{r}, y^{r}\right)$ such that $\rho^{l}>0, \rho^{r}>0, \rho^{l} \neq \rho^{r}$ but $v^{l}=v^{r}$. In this case, the solution simply consists in a contact discontinuity propagating at speed $v_{0}:=v^{l}=v^{r}$ :

$$
\mathbf{u}(x, t)=\left\{\begin{array}{c}
\mathbf{u}^{l} \text { if } x<v_{0} t \\
\mathbf{u}^{r} \text { if } x>v_{0} t
\end{array}\right.
$$

From now on, we assume $v_{0}>0$.

What happens in the first time step?

From (3.1) we have that

$$
\mathbf{u}_{j}^{0}=\left\{\begin{array}{c}
\mathbf{u}^{l} \text { if } j \leq 0, \\
\mathbf{u}^{r} \text { if } j>0 .
\end{array}\right.
$$

Due to the CFL restriction (3.4) and the property $v_{0}>0$, only the cell $\mathcal{C}_{1}$ may be affected by update formula (3.6) in the first time step. In other words,

$$
\mathbf{u}_{j}^{1}=\mathbf{u}_{j}^{0} \quad \text { for all } \quad j \neq 1 .
$$

For $j=1,(3.6)$ is equivalent to

$$
\rho_{1}^{1}=\bar{\rho} \quad \text { and } \quad y_{1}^{1}=\bar{y},
$$

where we have used the notation

$$
\bar{\alpha}=\frac{1}{\Delta x} \int_{0}^{\Delta x} \alpha(x, \Delta t) d x .
$$


We observe that

$$
\bar{y}=\frac{1}{\Delta x} \int_{0}^{\Delta x} y(x, \Delta t) d x=\frac{1}{\Delta x} \int_{0}^{\Delta x}(\rho v+\rho p(\rho))(x, \Delta t) d x .
$$

Since the velocity remains constant across a contact discontinuity, we have

$$
\bar{y}=v_{0} \frac{1}{\Delta x} \int_{0}^{\Delta x} \rho(x, \Delta t) d x+\frac{1}{\Delta x} \int_{0}^{\Delta x}(\rho p(\rho))(x, \Delta t) d x=v_{0} \bar{\rho}+\overline{\rho p(\rho)} .
$$

On the contrary, if we calculate $v_{1}^{1}$ from $\bar{\rho}$ and $\bar{y}$, we get

$$
v_{1}^{1}=\frac{\bar{y}}{\bar{\rho}}-p(\bar{\rho})=v_{0}+\frac{\overline{\rho p(\rho)}-\bar{\rho} p(\bar{\rho})}{\bar{\rho}} .
$$

We observe that the function $\rho \rightarrow \rho p(\rho)$ is convex. By Jensen's inequality, we deduce $\overline{\rho p(\rho)} \geq \bar{\rho} p(\bar{\rho})$ and then

$$
v_{1}^{1} \geq v_{0},
$$

with strict inequality generally speaking. Thus, after the first time iteration, the velocity no longer equals $v_{0}$ everywhere. We conclude that the Godunov method is not able to both keep the velocity profile constant and to capture properly contact discontinuities. In Section 4, we will show that the non-physical values created by the Godunov method around contact discontinuities may significantly damage the numerical solution. Our goal is to design a suitable algorithm to remove these spurious values.

REMARK 3.1.

(i) The failure we have just underscored is due to the fact that the Godunov method does not obey a maximum principle for the velocity $v$. The algorithm we propose in the next section verifies the maximum principle for the Riemann invariants $v$ and $v+p(\rho)$, see Theorem 3.2.

(ii) It is important to notice that if we now consider an isolated 1-wave between $\mathbf{u}^{l}$ and $\mathbf{u}^{r}$, the Godunov method actually keeps constant the Riemann invariant $v+p(\rho)$. If we set $C_{0}:=v^{l}+p\left(\rho^{l}\right)=v^{r}+p\left(\rho^{r}\right)$, we have indeed

$$
(v+p(\rho))_{1}^{1}=\left(\frac{y}{\rho}\right)_{1}^{1}=\frac{\bar{y}}{\bar{\rho}}=\frac{\overline{\rho(v+p(\rho))}}{\bar{\rho}}=C_{0} \frac{\bar{\rho}}{\bar{\rho}}=C_{0} .
$$

This property is very interesting and means in particular that all the points in a numerical 1-wave profile associated with Godunov's method belong to the same 1wave curve for all the possible choices of $p(\rho)$. This property is also satisfied by our new method (see Theorem 3.1 (iii) and Theorem 3.2).

3.3. A transport-equilibrium scheme. We propose now an algorithm that allows to avoid the spurious oscillations generated near the contact discontinuities by the classical Godunov method. The basic idea is to treat in a different way contact discontinuities on one side and other waves (shock and rarefaction waves) on the other side. We will keep on using the Godunov method for shocks and rarefactions (since it works well and is conservative), and we will propose a particular treatment for contact discontinuities which make use of a (Glimm) random sampling strategy. 


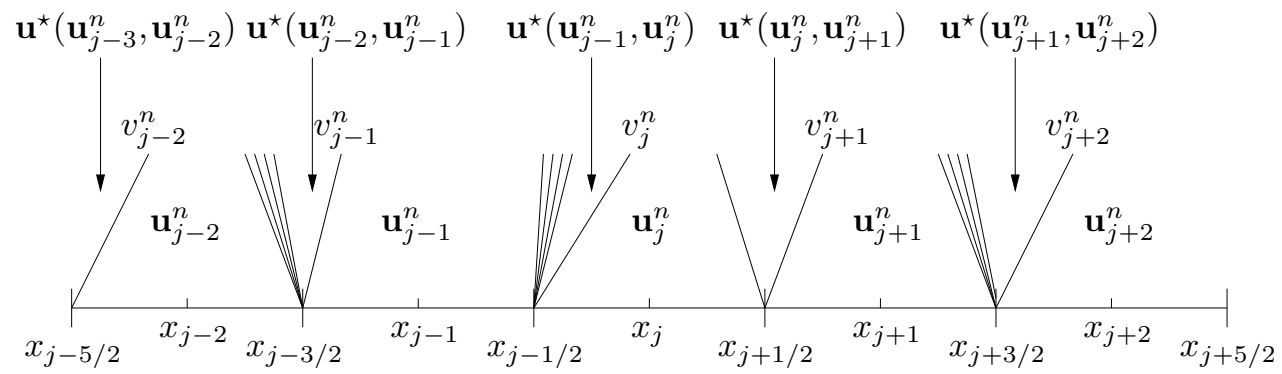

FIG. 3.1. Illustration of the notations used in the paper.

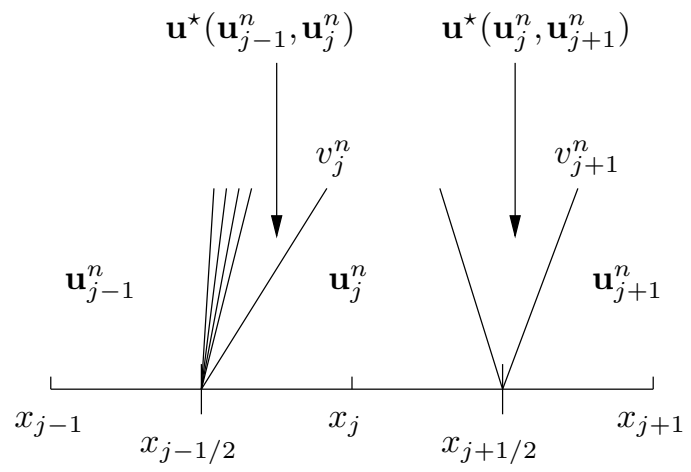

FIG. 3.2. Restriction on the interval $\left(x_{j-1}, x_{j+1}\right)$.

We set

$$
\mathbf{g}\left(\mathbf{u}^{l}, \mathbf{u}^{r}\right)=\mathbf{f}\left(\mathbf{u}_{\mathbf{r}}\left(0^{-} ; \mathbf{u}^{l}, \mathbf{u}^{r}\right)\right),
$$

so that the numerical flux of the Godunov method writes $\mathbf{f}_{j+1 / 2}^{n}=\mathbf{g}\left(\mathbf{u}_{j}^{n}, \mathbf{u}_{j+1}^{n}\right)$ for all $j \in \mathbb{Z}$. Recall that $\mathbf{u}^{\star}\left(\mathbf{u}^{l}, \mathbf{u}^{r}\right)$ is the intermediate state in the Riemann solution $\mathbf{u}_{\mathbf{r}}\left(. ; \mathbf{u}^{l}, \mathbf{u}^{r}\right)$ (between the 1-wave and the 2-contact discontinuity), so that $\mathbf{u}^{l}$ and $\mathbf{u}^{\star}\left(\mathbf{u}^{l}, \mathbf{u}^{r}\right)$ are connected by a 1-wave and $\mathbf{u}^{\star}\left(\mathbf{u}^{l}, \mathbf{u}^{r}\right)$ and $\mathbf{u}^{r}$ are connected by a 2 contact discontinuity. Of course, we have $\mathbf{u}^{\star}\left(\mathbf{u}^{l}, \mathbf{u}^{r}\right)=\mathbf{u}^{r}$ (respectively $\mathbf{u}^{\star}\left(\mathbf{u}^{l}, \mathbf{u}^{r}\right)=\mathbf{u}^{l}$ ) if $\mathbf{u}^{l}$ and $\mathbf{u}^{r}$ are connected by a 1-wave (respectively a 2-contact discontinuity), and we set $\mathbf{u}^{\star}(\mathbf{u}, \mathbf{u})=\mathbf{u}$ for all $\mathbf{u}$. See Fig. 3.1.

The method is composed of two steps. On each interval $\left[x_{j}, x_{j+1}\right], j \in \mathbb{Z}$, the first step takes into account only the contact discontinuity in the Riemann solution $\mathbf{u}_{\mathbf{r}}\left(. ; \mathbf{u}_{j}^{n}, \mathbf{u}_{j+1}^{n}\right)$, while the second step focuses on the 1-wave. Our procedure may be viewed as a waves-splitting strategy, performed locally around each interface $x_{j+1 / 2}$ where a Riemann problem is set.

Assuming as given $\mathbf{u}_{j-1}^{n}, \mathbf{u}_{j}^{n}$ and $\mathbf{u}_{j+1}^{n}$, we show now how to define $\mathbf{u}_{j}^{n+1}$. Note that, under the CFL condition (3.4), it is sufficient to focus on the interval $\left[x_{j-1}, x_{j+1}\right]$, since the Riemann problems set at other interfaces are not expected to influence the definition of $\mathbf{u}_{j}^{n+1}$. See below Fig. 3.2.

Step 1: Propagation of contact discontinuities $\left(t^{n} \rightarrow t^{n+1 / 2}\right)$.

In this step, we focus on the dynamics of contact discontinuities. We proceed as follows. The Riemann problems at interfaces $x_{j-1 / 2}$ and $x_{j+1 / 2}$ generally develop a 


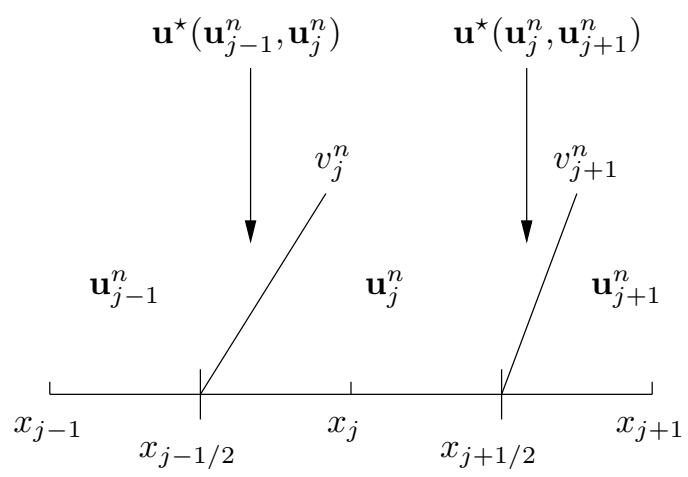

FIG. 3.3. Function $\tilde{\mathbf{v}}$.

1-wave and a 2-contact discontinuity, the latter propagating at speed $v_{j}^{n}$ and $v_{j+1}^{n}$ respectively (see again Fig. 3.2). These velocities being nonnegative, the contact discontinuities only affect $\left[x_{j-1 / 2}, x_{j}\right)$ and $\left[x_{j+1 / 2}, x_{j+1}\right]$, but not $\left[x_{j-1}, x_{j-1 / 2}\right)$ and $\left[x_{j}, x_{j+1 / 2}\right)$. This means that the Riemann solutions $\mathbf{u}_{\mathbf{r}}\left(. ; \mathbf{u}_{j-1}^{n}, \mathbf{u}_{j}^{n}\right)$ and $\mathbf{u}_{\mathbf{r}}\left(. ; \mathbf{u}_{j}^{n}, \mathbf{u}_{j+1}^{n}\right)$ could be replaced in this step with the following function

$$
\tilde{\mathbf{v}}(x, t)=\left\{\begin{aligned}
\mathbf{u}_{j-1}^{n} & \text { if } x \in\left[x_{j-1}, x_{j-1 / 2}\right) \\
\mathbf{u}^{\star}\left(\mathbf{u}_{j-1}^{n}, \mathbf{u}_{j}^{n}\right) & \text { if } x \in\left[x_{j-1 / 2}, x_{j-1 / 2}+v_{j}^{n}\left(t-t^{n}\right)\right) \\
\mathbf{u}_{j}^{n} & \text { if } x \in\left[x_{j-1 / 2}+v_{j}^{n}\left(t-t^{n}\right), x_{j+1 / 2}\right) \\
\mathbf{u}^{\star}\left(\mathbf{u}_{j}^{n}, \mathbf{u}_{j+1}^{n}\right) & \text { if } x \in\left[x_{j+1 / 2}, x_{j+1 / 2}+v_{j+1}^{n}\left(t-t^{n}\right)\right) \\
\mathbf{u}_{j+1}^{n} & \text { if } x \in\left[x_{j+1 / 2}+v_{j+1}^{n}\left(t-t^{n}\right), x_{j+1}\right]
\end{aligned}\right.
$$

on the whole interval $\left(x_{j-1}, x_{j+1}\right)$, see Fig. 3.3. Of course, this function has to be considered as a substitute of function $\mathbf{v}$ in (3.5), where only contact discontinuities have been kept.

In order to properly capture contact discontinuities, we propose to define $\tilde{\mathbf{v}}\left(x, t^{n+1 / 2}\right)$ as a piecewise constant function on each interval $\left[x_{j-1}, x_{j-1 / 2}\right)$, $\left[x_{j-1 / 2}, x_{j+1 / 2}\right)$ and $\left[x_{j+1 / 2}, x_{j+1}\right]\left(\right.$ as $\tilde{\mathbf{v}}\left(x, t^{n}\right)$ is) by means of Glimm's random sampling strategy. More precisely, we pick randomly on the cell $\left[x_{j-1}, x_{j+1}\right]$ a value between $\mathbf{u}_{j-1}^{n}, \mathbf{u}^{\star}\left(\mathbf{u}_{j-1}^{n}, \mathbf{u}_{j}^{n}\right), \mathbf{u}_{j}^{n}, \mathbf{u}^{\star}\left(\mathbf{u}_{j}^{n}, \mathbf{u}_{j+1}^{n}\right)$ and $\mathbf{u}_{j+1}^{n}$ in agreement with their degree of presence in the corresponding interval, or equivalently in agreement with the definition of the function $x \rightarrow \tilde{\mathbf{v}}\left(x, t^{n}+\Delta t\right)$. Given a well distributed random sequence $\left(a_{n}\right)$ within interval $(0,1)$, this amounts to setting:

$$
\tilde{\mathbf{v}}\left(x, t^{n+1 / 2}\right)=\left\{\begin{array}{cl}
\mathbf{u}_{j-1}^{n} & \text { if } x \in\left[x_{j-1}, x_{j-1 / 2}\right), \\
\mathbf{u}_{j}^{n+1 / 2} & \text { if } x \in\left[x_{j-1 / 2}, x_{j+1 / 2}\right), \\
\mathbf{u}_{j+1, L}^{n+1 / 2} & \text { if } x \in\left[x_{j+1 / 2}, x_{j+1}\right],
\end{array}\right.
$$

with

$$
\mathbf{u}_{j}^{n+1 / 2}=\left\{\begin{array}{cl}
\mathbf{u}^{\star}\left(\mathbf{u}_{j-1}^{n}, \mathbf{u}_{j}^{n}\right) & \text { if } a_{n+1} \in\left(0, \frac{\Delta t}{\Delta x} v_{j}^{n}\right), \\
\mathbf{u}_{j}^{n} & \text { if } a_{n+1} \in\left[\frac{\Delta t}{\Delta x} v_{j}^{n}, 1\right),
\end{array}\right.
$$

and

$$
\mathbf{u}_{j+1, L}^{n+1 / 2}=\left\{\begin{array}{cl}
\mathbf{u}^{\star}\left(\mathbf{u}_{j}^{n}, \mathbf{u}_{j+1}^{n}\right) & \text { if } a_{n+1} \in\left(0, \frac{2 \Delta t}{\Delta x} v_{j}^{n}\right), \\
\mathbf{u}_{j+1}^{n} & \text { if } a_{n+1} \in\left[\frac{2 \Delta t}{\Delta x} v_{j}^{n}, 1\right) .
\end{array}\right.
$$




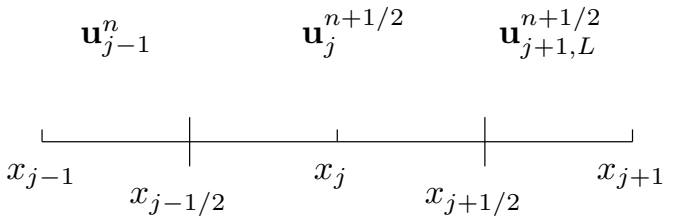

FIG. 3.4. Function $\tilde{\mathbf{v}}\left(x, t^{n+1 / 2}\right)$.

See Fig. 3.4. In practice, we will consider the well-known van der Corput random sequence $\left(a_{n}\right)$ defined by

$$
a_{n}=\sum_{k=0}^{m} i_{k} 2^{-(k+1)}
$$

where $n=\sum_{k=0}^{m} i_{k} 2^{k}, i_{k} \in\{0,1\}$ denotes the binary expansion of the integers $n=$ $1,2, \ldots$ (see for instance Collela [9]).

REMARK 3.2. It is worth noticing that if both $\mathbf{u}_{j-1}^{n}$ and $\mathbf{u}_{j}^{n}$ on one hand and $\mathbf{u}_{j}^{n}$, and $\mathbf{u}_{j+1}^{n}$ on the other hand can be connected by a 1-wave, then $\mathbf{u}^{\star}\left(\mathbf{u}_{j-1}^{n}, \mathbf{u}_{j}^{n}\right)=\mathbf{u}_{j}^{n}$ and $\mathbf{u}^{\star}\left(\mathbf{u}_{j}^{n}, \mathbf{u}_{j+1}^{n}\right)=\mathbf{u}_{j+1}^{n}$ and as an immediate consequence of $(3.10)-(3.11), \tilde{\mathbf{v}}\left(x, t^{n+1 / 2}\right)=$ $\tilde{\mathbf{v}}\left(x, t^{n}\right)$. So the first step is transparent when no contact discontinuity is present.

Step 2: Account for the dynamics of shock and rarefaction waves $\left(t^{n+1 / 2} \rightarrow t^{n+1}\right)$. Let us now concentrate on the parts of the solution located on the left and on the right of the contact discontinuities.

Let us first consider the Riemann problem set at the interface $x_{j+1 / 2}$ for which only the part of the solution located on the left of the contact discontinuity may enter the cell $\mathcal{C}_{j}=\left[x_{j-1 / 2}, x_{j+1 / 2}\right)$. See Fig. 3.2. We propose to take it into account by simply averaging $\mathbf{u}_{\mathbf{r}}\left(. ; \mathbf{u}_{j}^{n+1 / 2}, \mathbf{u}_{j+1, L}^{n+1 / 2}\right)$ on $\left(x_{j}, x_{j+1 / 2}\right)$ as in (3.8). Note that the restrictions to $\left[x_{j}, x_{j+1 / 2}\right)$ of $\mathbf{u}_{\mathbf{r}}\left(. ; \mathbf{u}_{j}^{n+1 / 2}, \mathbf{u}_{j+1, L}^{n+1 / 2}\right)$ and $\mathbf{u}_{\mathbf{r}}\left(. ; \mathbf{u}_{j}^{n+1 / 2}, \mathbf{u}_{j+1}^{n}\right)$ are the same since $\mathbf{u}_{j+1, L}^{n+1 / 2}$ and $\mathbf{u}_{j+1}^{n}$ are either equal or separated by a contact discontinuity. Then we set

$$
\begin{aligned}
\mathbf{u}_{j+1 / 2, L}^{n+1} & =\frac{2}{\Delta x} \int_{x_{j}}^{x_{j+1 / 2}} \mathbf{u}_{\mathbf{r}}\left(\frac{x-x_{j+1 / 2}}{\Delta t} ; \mathbf{u}_{j}^{n+1 / 2}, \mathbf{u}_{j+1}^{n}\right) d x \\
& =\mathbf{u}_{j}^{n+1 / 2}-\frac{2 \Delta t}{\Delta x}\left(\mathbf{g}\left(\mathbf{u}_{j}^{n+1 / 2}, \mathbf{u}_{j+1}^{n}\right)-\mathbf{f}\left(\mathbf{u}_{j}^{n+1 / 2}\right)\right) .
\end{aligned}
$$

Let us now focus on the Riemann problem set at the interface $x_{j-1 / 2}$ for which both parts of the solution located on the left and on the right of the contact discontinuity may enter the cell $\mathcal{C}_{j}$ depending on the sense of propagation of the 1 -wave (see again Fig. 3.2). There are two possibilities:

- $\mathbf{u}_{j}^{n+1 / 2}=\mathbf{u}_{j}^{n}$. This corresponds to the situation where the random sampling "decided" that the (possibly present) contact discontinuity of $\mathbf{u}_{\mathbf{r}}\left(. ; \mathbf{u}_{j-1}^{n}, \mathbf{u}_{j}^{n}\right)$ has not yet entered the cell $\mathcal{C}_{j}$. Then we only have to account for:

- the right part of the contact discontinuity in $\mathbf{u}_{\mathbf{r}}\left(. ; \mathbf{u}_{j-1}^{n}, \mathbf{u}_{j}^{n}\right)$ (i.e. $\mathbf{u}_{j}^{n}=$ $\left.\mathbf{u}_{j}^{n+1 / 2}\right)$ if a contact discontinuity is actually present in $\mathbf{u}_{\mathbf{r}}\left(. ; \mathbf{u}_{j-1}^{n}, \mathbf{u}_{j}^{n}\right)$, that is if $\mathbf{u}^{\star}\left(\mathbf{u}_{j-1}^{n}, \mathbf{u}_{j}^{n}\right) \neq \mathbf{u}_{j}^{n}$. This is simply done by replacing $\mathbf{u}_{j-1}^{n}$ and $\mathbf{u}_{j}^{n}$ with $\mathbf{u}_{j}^{n+1 / 2}=\mathbf{u}_{j}^{n}$ in $\mathbf{u}_{\mathbf{r}}\left(. ; \mathbf{u}_{j-1}^{n}, \mathbf{u}_{j}^{n}\right)$; 
- the part of the solution $\mathbf{u}_{\mathbf{r}}\left(. ; \mathbf{u}_{j-1}^{n}, \mathbf{u}_{j}^{n}\right)$ entering $\mathcal{C}_{j}$ if no contact discontinuity is present in $\mathbf{u}_{\mathbf{r}}\left(. ; \mathbf{u}_{j-1}^{n}, \mathbf{u}_{j}^{n}\right)$, that is if $\mathbf{u}^{\star}\left(\mathbf{u}_{j-1}^{n}, \mathbf{u}_{j}^{n}\right)=\mathbf{u}_{j}^{n}$.

- $\mathbf{u}_{j}^{n+1 / 2}=\mathbf{u}^{\star}\left(\mathbf{u}_{j-1}^{n}, \mathbf{u}_{j}^{n}\right) \neq \mathbf{u}_{j}^{n}$. This corresponds to the situation where the random sampling decided to make the contact discontinuity of $\mathbf{u}_{\mathbf{r}}\left(. ; \mathbf{u}_{j-1}^{n}, \mathbf{u}_{j}^{n}\right)$ enter the cell $\mathcal{C}_{j}$. Then we also have to account for the part of the solution $\mathbf{u}_{\mathbf{r}}\left(. ; \mathbf{u}_{j-1}^{n}, \mathbf{u}_{j}^{n}\right)$ located on the left of the contact discontinuity and entering the cell $\mathcal{C}_{j}$, equivalently the part of the solution $\mathbf{u}_{\mathbf{r}}\left(. ; \mathbf{u}_{j-1}^{n}, \mathbf{u}_{j}^{n+1 / 2}\right)$ entering the cell $\mathcal{C}_{j}$.

Thus, since the condition $\mathbf{u}^{\star}\left(\mathbf{u}_{j-1}^{n}, \mathbf{u}_{j}^{n}\right) \neq \mathbf{u}_{j}^{n}$ is equivalent to $\mathbf{u}^{\star}\left(\mathbf{u}_{j-1}^{n}, \mathbf{u}_{j}^{n+1 / 2}\right) \neq$ $\mathbf{u}_{j}^{n+1 / 2}$ when $\mathbf{u}_{j}^{n+1 / 2}=\mathbf{u}_{j}^{n}$, and by averaging on $\left[x_{j-1 / 2}, x_{j}\right)$ as in (3.9), we are led to set

$$
\begin{aligned}
\mathbf{u}_{j-1 / 2, R}^{n+1} & =\frac{2}{\Delta x} \int_{x_{j-1 / 2}}^{x_{j}} \mathbf{u}_{\mathbf{r}}\left(\frac{x-x_{j-1 / 2}}{\Delta t} ; \mathbf{u}_{j-1}^{n}, \mathbf{u}_{j}^{n+1 / 2}\right) d x \\
& =\mathbf{u}_{j}^{n+1 / 2}-\frac{2 \Delta t}{\Delta x}\left(\mathbf{f}\left(\mathbf{u}_{j}^{n+1 / 2}\right)-\mathbf{g}\left(\mathbf{u}_{j-1}^{n}, \mathbf{u}_{j}^{n+1 / 2}\right)\right) \\
\text { if } \quad \mathbf{u}^{\star}\left(\mathbf{u}_{j-1}^{n}, \mathbf{u}_{j}^{n+1 / 2}\right) & =\mathbf{u}_{j}^{n+1 / 2}, \\
\mathbf{u}_{j-1 / 2, R}^{n+1} & =\frac{2}{\Delta x} \int_{x_{j-1 / 2}}^{x_{j}} \mathbf{u}_{\mathbf{r}}\left(\frac{x-x_{j-1 / 2}}{\Delta t} ; \mathbf{u}_{j}^{n+1 / 2}, \mathbf{u}_{j}^{n+1 / 2}\right) d x=\mathbf{u}_{j}^{n+1 / 2}
\end{aligned}
$$

otherwise.

By (3.7), we get the following update formula:

$$
\mathbf{u}_{j}^{n+1}=\frac{1}{2}\left(\mathbf{u}_{j+1 / 2, L}^{n+1}+\mathbf{u}_{j-1 / 2, R}^{n+1}\right)=\mathbf{u}_{j}^{n+1 / 2}-\frac{\Delta t}{\Delta x}\left(\mathbf{g}_{j+1 / 2}^{n+1 / 2, L}-\mathbf{g}_{j-1 / 2}^{n+1 / 2, R}\right) \text { for all } j \in \mathbb{Z},
$$

where the left and right numerical flux functions $\mathbf{g}_{j+1 / 2}^{n+1 / 2, L}$ and $\mathbf{g}_{j-1 / 2}^{n+1 / 2, R}$ are defined according to

$$
\begin{aligned}
& \mathbf{g}_{j+1 / 2}^{n+1 / 2, L}=\mathbf{g}\left(\mathbf{u}_{j}^{n+1 / 2}, \mathbf{u}_{j+1}^{n}\right),
\end{aligned}
$$

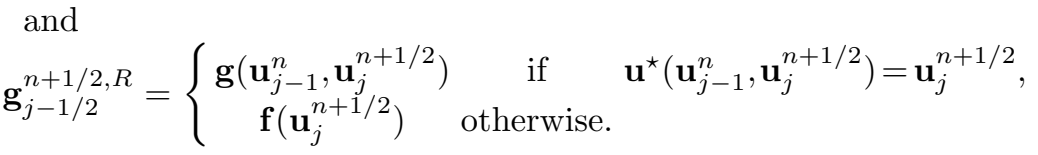

The description of the method is now completed. Stability properties enjoyed by this algorithm are proposed below.

REMARK 3.3. Putting the first and the second step together, we note that the definition of $\mathbf{u}_{j}^{n+1}$ only depends on $\mathbf{u}_{j-1}^{n}, \mathbf{u}_{j}^{n}$ and $\mathbf{u}_{j+1}^{n}$.

REMARK 3.4. For numerical reasons, the test $\mathbf{u}^{\star}\left(\mathbf{u}_{j}^{n}, \mathbf{u}_{j+1}^{n+1 / 2}\right)=\mathbf{u}_{j+1}^{n+1 / 2}$ in (3.15) is replaced with $\left|\mathbf{u}^{\star}\left(\mathbf{u}_{j}^{n}, \mathbf{u}_{j+1}^{n+1 / 2}\right)-\mathbf{u}_{j+1}^{n+1 / 2}\right| \leq \epsilon$, with for instance $\epsilon=10^{-12}$.

Theorem 3.1. (Consistency). Under the CFL restriction (3.4), the scheme defined by (3.10)-(3.14)-(3.15) is consistent with (1.5) in the following sense:

(i) Constant state: Assume that $\mathbf{u}:=\mathbf{u}_{j-1}^{n}=\mathbf{u}_{j}^{n}=\mathbf{u}_{j+1}^{n}$. Then $\mathbf{u}_{j}^{n+1}=\mathbf{u}$.

(ii) Isolated contact discontinuity: Let $\mathbf{u}^{l}$ and $\mathbf{u}^{r}$ be two distinct constant states that can be connected by a contact discontinuity. We set $v:=v^{l}=v^{r}$. Assume that 
$\mathbf{u}_{j}^{0}=\mathbf{u}^{l}$ if $j \leq 0$ and $\mathbf{u}_{j}^{0}=\mathbf{u}^{r}$ if $j>0$. Then the scheme (3.10)-(3.14)-(3.15) is equivalent to Glimm's random choice scheme and converges to the solution of (2.2) given by $\mathbf{u}(x, t)=\mathbf{u}^{l}$ if $x<v t$ and $\mathbf{u}(x, t)=\mathbf{u}^{r}$ otherwise. In particular, we have $\mathbf{u}_{j}^{n} \in\left\{\mathbf{u}^{l}, \mathbf{u}^{r}\right\}$ $\forall j \in \mathbb{Z}$ and $\forall n \in \mathbb{N}$ so that the velocity profile remains constant: $v_{j}^{n}=v \forall j \in \mathbb{Z}$ and $\forall n \in \mathbb{N}$.

(iii) Isolated 1-wave: Let us assume that $\mathbf{u}_{j-1}^{n}$ and $\mathbf{u}_{j}^{n}$ can be connected by a 1-wave $\left(\mathbf{u}^{\star}\left(\mathbf{u}_{j-1}^{n}, \mathbf{u}_{j}^{n}\right)=\mathbf{u}_{j}^{n}\right)$. Then the definition $\mathbf{u}_{j}^{n+1}$ of our scheme (3.10)-(3.14)(3.15) coincides with the definition of the Godunov scheme, that is, $\mathbf{u}_{j}^{n+1}$ is given by (3.2).

Proof.

(i) Let us assume that $\mathbf{u}_{l}^{n}=\mathbf{u}$ for $l=j-1, j, j+1$. Then, $\mathbf{u}^{\star}\left(\mathbf{u}_{j-1}^{n}, \mathbf{u}_{j}^{n}\right)=\mathbf{u}^{\star}(\mathbf{u}, \mathbf{u})=$ $\mathbf{u}$ and, by formula (3.10), we necessarily have $\mathbf{u}_{j}^{n+1 / 2}=\mathbf{u}$. Recall now the consistency property $\mathbf{g}(\mathbf{u}, \mathbf{u})=\mathbf{f}(\mathbf{u})$ of the Godunov flux function. Invoking definition (3.15), we thus have

$$
\mathbf{g}_{j+1 / 2}^{n+1 / 2, L}=\mathbf{g}(\mathbf{u}, \mathbf{u})=\mathbf{f}(\mathbf{u}) \quad \text { and } \quad \mathbf{g}_{j+1 / 2}^{n+1 / 2, R}=\mathbf{g}(\mathbf{u}, \mathbf{u})=\mathbf{f}(\mathbf{u})
$$

Then, formula (3.14) proves that $\mathbf{u}_{j}^{n+1}=\mathbf{u}$.

(ii) Let us set $\mathbf{u}_{j}^{0}=\mathbf{u}^{l}$ if $j \leq 0$ and $\mathbf{u}_{j}^{0}=\mathbf{u}^{r}$ if $j>0$. Since $\mathbf{u}^{l}$ and $\mathbf{u}^{r}$ are assumed to be connected by a contact discontinuity, we have in particular $\mathbf{u}^{\star}\left(\mathbf{u}^{l}, \mathbf{u}^{r}\right)=\mathbf{u}^{l}$ (while of course $\mathbf{u}^{\star}\left(\mathbf{u}^{l}, \mathbf{u}^{l}\right)=\mathbf{u}^{l}$ and $\left.\mathbf{u}^{\star}\left(\mathbf{u}^{r}, \mathbf{u}^{r}\right)=\mathbf{u}^{r}\right)$ so that the definition of $\mathbf{u}_{j}^{n+1 / 2}$ in (3.10) coincides with the definition provided by the Glimm scheme. To prove our result, we thus have to show that $\mathbf{u}_{j}^{n+1}=\mathbf{u}_{j}^{n+1 / 2}$. The two situations of interest are first $\mathbf{u}_{k}^{n+1 / 2}=\mathbf{u}^{l}$ for $k \leq j$ and $\mathbf{u}_{k}^{n+1 / 2}=\mathbf{u}^{r}$ otherwise, and second $\mathbf{u}_{k}^{n+1 / 2}=\mathbf{u}^{l}$ for $k<j$ and $\mathbf{u}_{k}^{n+1 / 2}=\mathbf{u}^{r}$ otherwise.

In the first situation, we necessarily have $\mathbf{u}_{j-1}^{n}=\mathbf{u}^{l}$ (respectively $\mathbf{u}_{j+1}^{n}=\mathbf{u}^{r}$ ), since otherwise we would have $\mathbf{u}_{j-1}^{n}=\mathbf{u}_{j}^{n}=\mathbf{u}^{r}$ and then $\mathbf{u}_{j}^{n+1 / 2}=\mathbf{u}^{r}$ (respectively $\mathbf{u}_{j}^{n}=$ $\mathbf{u}_{j+1}^{n}=\mathbf{u}^{l}$ and then $\left.\mathbf{u}_{j+1}^{n+1 / 2}=\mathbf{u}^{l}\right)$. Then we have $\mathbf{g}_{j+1 / 2}^{n+1 / 2, L}=\mathbf{g}\left(\mathbf{u}^{l}, \mathbf{u}^{r}\right)=\mathbf{f}\left(\mathbf{u}^{l}\right)$, because the contact discontinuity propagates to the right-hand side. Moreover, $\mathbf{g}_{j-1 / 2}^{n+1 / 2, R}=$ $\mathbf{g}\left(\mathbf{u}^{l}, \mathbf{u}^{l}\right)=\mathbf{f}\left(\mathbf{u}^{l}\right)$, so that $\mathbf{u}_{j}^{n+1}=\mathbf{u}_{j}^{n+1 / 2}$ by $(3.14)$.

In the second situation, we note that $\mathbf{u}_{j+1}^{n}=\mathbf{u}^{r}$, since otherwise we would have $\mathbf{u}_{j}^{n}=\mathbf{u}_{j+1}^{n}=\mathbf{u}^{l}$ and then $\mathbf{u}_{j}^{n+1 / 2}=\mathbf{u}^{l}$. Hence $\mathbf{g}_{j+1 / 2}^{n+1 / 2, L}=\mathbf{g}\left(\mathbf{u}^{r}, \mathbf{u}^{r}\right)=\mathbf{f}\left(\mathbf{u}^{r}\right)$. As far as $\mathbf{g}_{j-1 / 2}^{n+1 / 2, R}$ is concerned, either

- $\mathbf{u}_{j-1}^{n}=\mathbf{u}^{l}$ and then $\mathbf{u}^{\star}\left(\mathbf{u}_{j-1}^{n}, \mathbf{u}_{j}^{n+1 / 2}\right)=\mathbf{u}^{\star}\left(\mathbf{u}^{l}, \mathbf{u}^{r}\right)=\mathbf{u}^{l} \neq \mathbf{u}^{r}$, which means $\mathbf{g}_{j-1 / 2}^{n+1 / 2, R}=\mathbf{f}\left(\mathbf{u}^{r}\right)$ by $(3.15)$, or

- $\mathbf{u}_{j-1}^{n}=\mathbf{u}^{r}$ and then $\mathbf{u}^{\star}\left(\mathbf{u}_{j-1}^{n}, \mathbf{u}_{j}^{n+1 / 2}\right)=\mathbf{u}^{\star}\left(\mathbf{u}^{r}, \mathbf{u}^{r}\right)=\mathbf{u}^{r}$, which means again $\mathbf{g}_{j-1 / 2}^{n+1 / 2, R}=\mathbf{f}\left(\mathbf{u}^{r}\right)$ by $(3.15)$. follows.

The two fluxes $\mathbf{g}_{j+1 / 2}^{n+1 / 2, L}$ and $\mathbf{g}_{j-1 / 2}^{n+1 / 2, R}$ then coincide and equality $\mathbf{u}_{j}^{n+1}=\mathbf{u}_{j}^{n+1 / 2}$

We have thus proved that the second step of the algorithm is transparent, which means that the method reduces to the first step and it is equivalent to Glimm's random choice scheme. It is proved in [18] (see also [22]) that the solution $\mathbf{u}(x, t)=\mathbf{u}^{l}$ for $x<v t$ and $\mathbf{u}(x, t)=\mathbf{u}^{r}$ for $x>v t$ converges if the random sequence $\left(a_{n}\right)$ is well distributed. 
(iii) If $\mathbf{u}_{j-1}^{n}$ and $\mathbf{u}_{j}^{n}$ can be connected by a 1-wave, we have $\mathbf{u}^{\star}\left(\mathbf{u}_{j-1}^{n}, \mathbf{u}_{j}^{n}\right)=\mathbf{u}_{j}^{n}$ and then $\mathbf{u}_{j}^{n+1 / 2}=\mathbf{u}_{j}^{n}$. Hence, by definition (3.15), we have $\mathbf{g}_{j+1 / 2}^{n+1 / 2, L}=\mathbf{g}\left(\mathbf{u}_{j}^{n}, \mathbf{u}_{j+1}^{n}\right)=\mathbf{f}_{j+1 / 2}^{n}$ and $\mathbf{g}_{j-1 / 2}^{n+1 / 2, R}=\mathbf{g}\left(\mathbf{u}_{j-1}^{n}, \mathbf{u}_{j}^{n}\right)=\mathbf{f}_{j-1 / 2}^{n}$. Then the definition of $\mathbf{u}_{j}^{n+1}$ given by our scheme (3.10)-(3.14)-(3.15) coincides with the one of the Godunov scheme, that is, $\mathbf{u}_{j}^{n+1}$ is given by (3.2). This completes the proof of the theorem.

THEOREM 3.2. (Maximum principle). Under the CFL restriction (3.4), the scheme defined by (3.10)-(3.14)-(3.15) satisfies the following maximum principle for all $j \in \mathbb{Z}$ and all $n \in \mathbb{N}$ :

$$
\left\{\begin{array}{l}
\inf _{j \in \mathbb{Z}} v_{j}^{0} \leq v_{j}^{n} \leq \sup _{j \in \mathbb{Z}} v_{j}^{0} \\
\inf _{j \in \mathbb{Z}}\left(v_{j}^{0}+p\left(\rho_{j}^{0}\right)\right) \leq v_{j}^{n}+p\left(\rho_{j}^{n}\right) \leq \sup _{j \in \mathbb{Z}}\left(v_{j}^{0}+p\left(\rho_{j}^{0}\right)\right) .
\end{array}\right.
$$

Proof. The maximum principle is not affected by the first step since for all $j \in \mathbb{Z}$, $\mathbf{u}_{j}^{n+1 / 2}$ is defined by picking up randomly a value between $\mathbf{u}^{\star}\left(\mathbf{u}_{j-1}^{n}, \mathbf{u}_{j}^{n}\right)$ and $\mathbf{u}_{j}^{n}$. Since in each Riemann solution one has $v+p(\rho)=$ const and $v=$ const along the first wave and the second wave respectively, we obtain

$$
\inf \left\{v_{j-1}^{n}+p\left(\rho_{j-1}^{n}\right), v_{j}^{n}+p\left(\rho_{j}^{n}\right)\right\} \leq v_{j}^{n+1 / 2}+p\left(\rho_{j}^{n+1 / 2}\right) \leq \sup \left\{v_{j-1}^{n}+p\left(\rho_{j-1}^{n}\right), v_{j}^{n}+p\left(\rho_{j}^{n}\right)\right\}
$$

and

$$
v_{j}^{n+1 / 2}=v_{j}^{n}
$$

Concerning the second step, new values are introduced in cells $\mathcal{C}_{j}$ by taking averages of (parts of) Riemann solutions which contain only waves of the first family, namely, $\mathbf{u}_{\mathbf{r}}\left(. ; \mathbf{u}_{j}^{n+1 / 2}, \mathbf{u}_{j+1}^{n}\right)$ on $\left[x_{j}, x_{j+1 / 2}\right)$, and $\mathbf{u}_{\mathbf{r}}\left(. ; \mathbf{u}_{j-1}^{n}, \mathbf{u}_{j}^{n+1 / 2}\right)$ or $\mathbf{u}_{\mathbf{r}}\left(. ; \mathbf{u}_{j}^{n+1 / 2}, \mathbf{u}_{j}^{n+1 / 2}\right)$ on $\left[x_{j-1 / 2}, x_{j}\right)$. Since again $v+p(\rho)=$ const $=C$ along these waves, and therefore $y=$ $\rho C$, we get $y_{j}^{n+1}=\rho_{j}^{n+1} C_{j}^{n+1 / 2}=\rho_{j}^{n+1}\left(v_{j}^{n+1 / 2}+p\left(\rho_{j}^{n+1 / 2}\right)\right)$, so that $v_{j}^{n+1}+p\left(\rho_{j}^{n+1}\right)=$ $C_{j}^{n+1 / 2}=v_{j}^{n+1 / 2}+p\left(\rho_{j}^{n+1 / 2}\right)$. The maximum principle thus holds for $v+p(\rho)$.

We now calculate the velocity $v_{j}^{n+1}$ using formula

$$
v_{j}^{n+1}=\frac{y_{j}^{n+1}}{\rho_{j}^{n+1}}-p\left(\rho_{j}^{n+1}\right)=C_{j}^{n+1 / 2}-p\left(\rho_{j}^{n+1}\right) .
$$

Let us denote by $\rho^{-}$(respectively $\rho^{+}$) the minimum (respectively maximum) value of $\rho$ among all the states involved in the averaging procedure of the second step and by $v^{-}$(respectively $v^{+}$) the corresponding velocity. Since the function $p$ is monotone (increasing), we get

$$
\inf \left\{v_{j-1}^{n}, v_{j}^{n+1 / 2}=v_{j}^{n}, v_{j+1}^{n}\right\} \leq v^{+} \leq v_{j}^{n+1} \leq v^{-} \leq \sup \left\{v_{j-1}^{n}, v_{j}^{n+1 / 2}=v_{j}^{n}, v_{j+1}^{n}\right\},
$$

so the maximum principle also holds for $v$.

REMARK 3.5. The present algorithm is consistent with (1.5) in the sense of Theorem 3.1 and obeys a maximum principle for both Riemann invariants $v+p(\rho)$ and $v$. By the latter property, contact discontinuities are properly computed (without oscillations), as is clearly shown in the next section. The counterpart is that our method no longer 
conserves the density and the momentum, even if the conservation errors are very small (see again the next section). Instead of averaging $\rho$ and $y$ as in the usual Godunov method, a way to properly capture the contact discontinuities while still conserving $\rho$ would be to average the density $\rho$ and the velocity $v$. However, the difficulty would be to obtain a simple form of the corresponding update formula, since $v$ is not a conservative variable for general pressure laws, as well as a maximum principle on $v+p(\rho)$.

REMARK 3.6. (Computational cost). According to (3.14), the definition of $\mathbf{u}_{j}^{n+1}$ relies on the computation of the time-intermediate value $\mathbf{u}_{j}^{n+1 / 2}$ and two numerical fluxes $\mathbf{g}_{j+1 / 2}^{n+1 / 2, L}$ and $\mathbf{g}_{j-1 / 2}^{n+1 / 2, R}$. The computation of $\mathbf{u}_{j}^{n+1 / 2}$ is generally associated with the computation of $\mathbf{u}^{\star}\left(\mathbf{u}_{j-1}^{n}, \mathbf{u}_{j}^{n}\right)$ by (3.10), while the computation of $\mathbf{g}_{j+1 / 2}^{n+1 / 2, L}$ and $\mathbf{g}_{j-1 / 2}^{n+1 / 2, R}$ corresponds in general to the computation of two Godunov fluxes by (3.15), namely $\mathbf{g}\left(\mathbf{u}_{j}^{n+1 / 2}, \mathbf{u}_{j+1}^{n}\right)$ and $\mathbf{g}\left(\mathbf{u}_{j-1}^{n}, \mathbf{u}_{j}^{n+1 / 2}\right)$. It is clear that the computation of $\mathbf{u}^{\star}\left(\mathbf{u}_{j-1}^{n}, \mathbf{u}_{j}^{n}\right)$ is part of the computation of the Godunov flux $\mathbf{g}\left(\mathbf{u}_{j}^{n+1 / 2}, \mathbf{u}_{j+1}^{n}\right)$. Hence the definition of $\mathbf{u}_{j}^{n+1}$ is eventually associated with the computation of two Godunov fluxes instead of one as in the usual Godunov method (since it is conservative). This additional cost is usual when trying to properly compute contact discontinuities, see [2] for instance. However, the computation of a Godunov flux is not expensive for the system under consideration in this paper (see Section 2).

\section{Numerical experiments}

Let us first recall that in this section, $p$ is given definition (1.3). We will take $R=1$ and $v_{r e f}=1.4427$. In order to test the proposed scheme, we consider three Riemann problems leading to three solutions of interest: an isolated contact discontinuity (Test 1), a shock wave followed by a contact discontinuity (Test 2) and a sonic rarefaction wave followed by a contact discontinuity (Test 3). In each case, the method is first evaluated by means of a qualitative comparison with the exact solution: the $\rho, v$ and $v+p(\rho)$ profiles are shown on figures 5.1, 5.2 and 5.3. For several mesh sizes, a quantitative evaluation through the $L^{1}$-norm (of the difference between the exact and numerical solutions) is then made, as well as a measure of the conservation errors on both $\rho$ and $y$. They are given on tables 5.1, 5.2, 5.3: $E_{\text {cons }}^{\rho}$ and $E_{\text {cons }}^{y}$ denote the conservation errors on $\rho$ and $y$, and $E_{L^{1}}^{\rho}$ and $E_{L^{1}}^{v}$ denote the $L^{1}$-errors on $\rho$ and $v$. The $L^{1}$-norm errors are computed in a very classical way. For the sake of completeness, we now give the precise meaning of $E_{\text {cons }}^{\rho}$ and $E_{c o n s}^{y}$ in our computations (and we refer the reader to $[2,5,6]$,for instance, for more details on these formulas): denoting by $\left[x_{0}, x_{1}\right]=[-0.25,0.75]$ the computational domain of our simulations and by $T_{f}$ the corresponding final time, we first set for all $n \geq 0$

$$
\begin{aligned}
E^{\mathbf{u}}\left(t^{n}\right) & =\left(\begin{array}{c}
E^{\rho}\left(t^{n}\right) \\
E^{y}\left(t^{n}\right)
\end{array}\right) \\
& =\frac{\int_{x_{0}}^{x_{1}} \mathbf{u}_{\nu}\left(x, t^{n}\right) d x-\int_{x_{0}}^{x_{1}} \mathbf{u}_{\nu}(x, 0) d x+\int_{0}^{t^{n}} \mathbf{f}\left(\mathbf{u}_{\nu}\left(x_{1}, s\right)\right) d s-\int_{0}^{t^{n}} \mathbf{f}\left(\mathbf{u}_{\nu}\left(x_{0}, s\right)\right) d s}{\int_{x_{0}}^{x_{1}} \mathbf{u}_{\nu}\left(x, t^{n}\right) d x}
\end{aligned}
$$

where the ratio is to be understood component by component, and then 


$$
E_{\text {cons }}^{\mathbf{u}}=\left(\begin{array}{l}
E_{\text {cons }}^{\rho} \\
E_{\text {cons }}^{y}
\end{array}\right)=\frac{1}{T_{f}} \sum_{n=0}^{N} \Delta t\left|E^{\mathbf{u}}\left(t^{n}\right)\right| \quad \text { with } N=T_{f} / \Delta t .
$$

Note that since $\mathbf{u}_{\nu}$ is piecewise constant, these quantities are easy to evaluate. Note also that $E_{\text {cons }}^{\mathbf{u}}$ corresponds to the sum of the absolute value of the relative conservation errors made at each intermediate time $t^{n}$. In other words, the possible compensation effects are not taken into account here.

Initial states are chosen as follows:

Test 1

Test 2

Test 3

$\begin{array}{ccccccccc}\mathbf{u}^{l}: & \rho^{l}=0.9 & v^{l}=1 . & \mathbf{u}^{l}: & \rho^{l}=0.1 & v^{l}=1.8 & \mathbf{u}^{l}: & \rho^{l}=0.5 & v^{l}=1.2 \\ \mathbf{u}^{r}: & \rho^{r}=0.1 & v^{r}=1 . & \mathbf{u}^{r}: & \rho^{r}=0.2 & v^{r}=1.6 & \mathbf{u}^{r}: & \rho^{r}=0.1 & v^{r}=1.6\end{array}$

The qualitative results are presented on a mesh made of 100 points per unit interval.

We observe as predicted above that the classical Godunov method develops spurious oscillations near the contact discontinuity that strongly affect the whole numerical solution. ${ }^{1}$ In contrast, our algorithm removes them and provides numerical solutions in full agreement with exact ones, and with sharp contact discontinuities. The conservation errors and the $L^{1}$-errors tend to zero with the mesh size, which proves numerically the convergence of the method. Moreover, we see that the $L^{1}$-errors between the numerical and exact solutions are actually lower for our scheme than for the Godunov method.

\section{Conclusion}

We have presented an algorithm whose objective is to remove the spurious oscillations generated by the Godunov scheme near contact discontinuities in the Aw-Rascle model for traffic flow. The method proposes to treat separately the contact discontinuities using a random sampling strategy, and to retain the Godunov scheme for the other waves. The whole algorithm is not strictly conservative but numerical experiments give very good numerical solutions with sharp (i.e. without numerical diffusion nor oscillations) contact discontinuities and small conservation errors, and show the numerical convergence. Moreover, we are able to prove that the method enjoys important stability properties like strong consistency and the maximum principle for the Riemann invariants of the system.

The resulting scheme remains dependent on the Riemann solver being exact. Even though it is very cheap in the present context, it would be interesting to observe how our scheme behaves when the exact Riemann solver is replaced by an approximate Riemann solver.

Another important topic would be the extension of the presented approach to higher-order schemes.

\footnotetext{
${ }^{1}$ We also observe that the spurious oscillations are mainly located behind the contact discontinuity. The reason which can explain this fact is probably that, in any given Riemann problem, the wave which propagates with the largest velocity is precisely the contact discontinuity (the other waves are then located behind the contact discontinuity).
} 

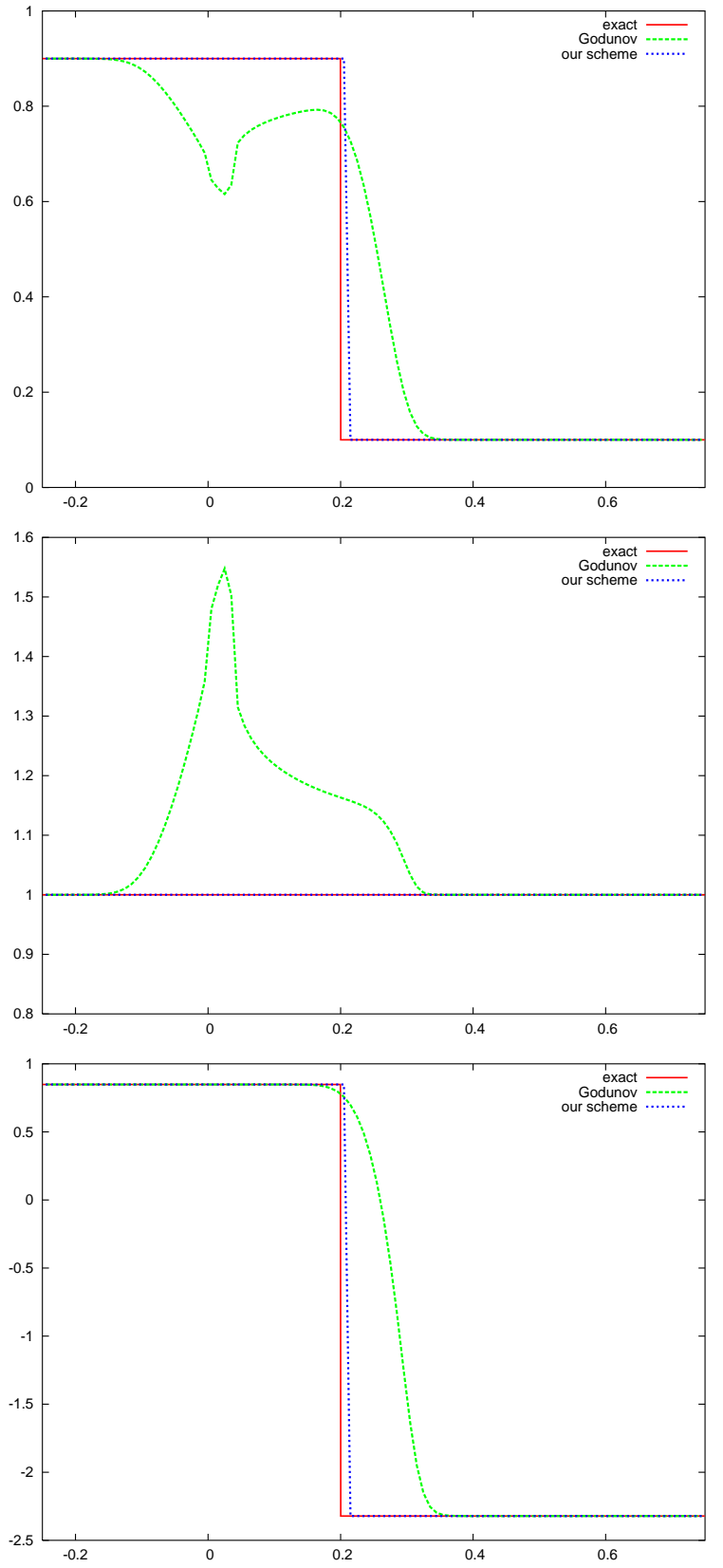

FIG. 5.1. Test 1: $\rho$ (Left), $v$ (Right) and $v+p(\rho)$ (Bottom) at time $t=0.2$. 

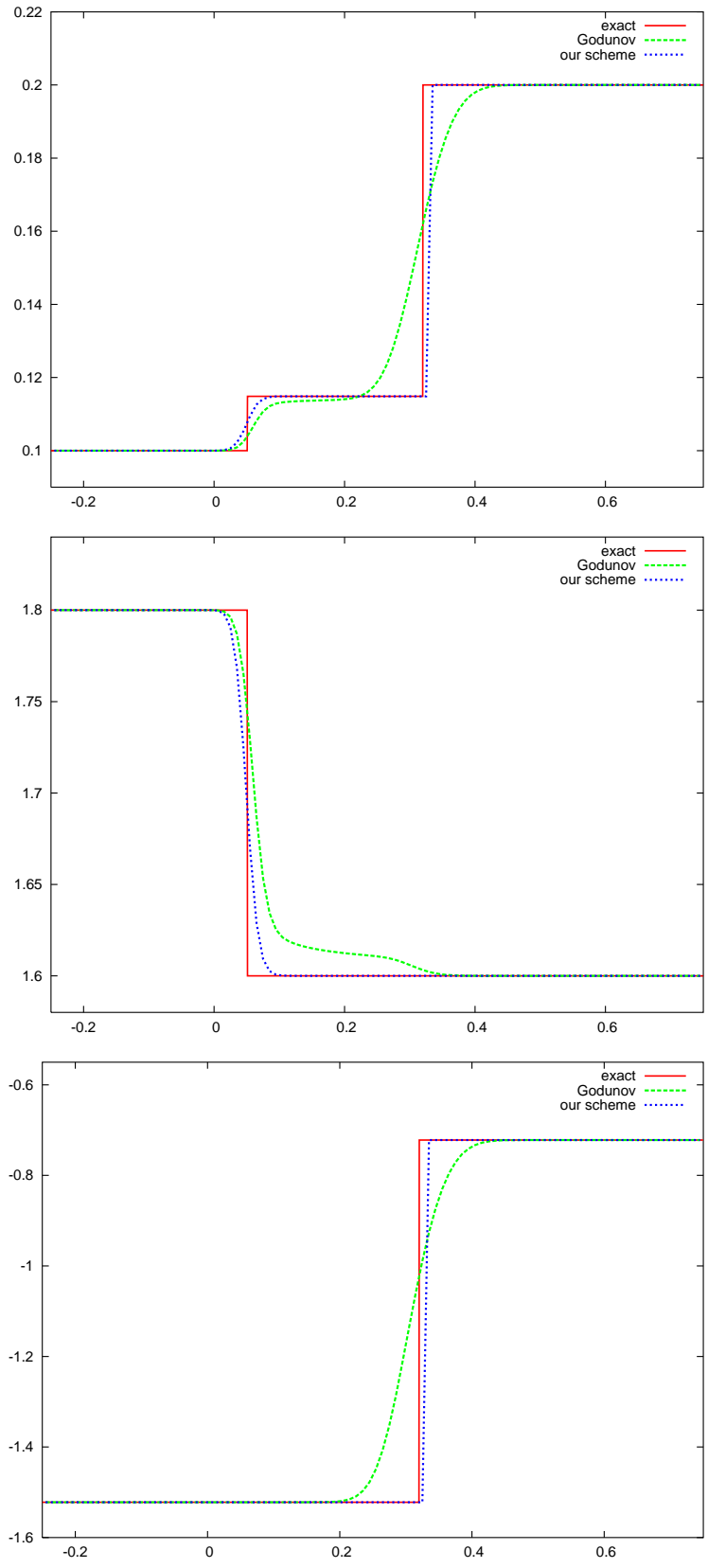

FIG. 5.2. Test 2: $\rho$ (Left), $v$ (Right) and $v+p(\rho)$ (Bottom) at time $t=0.2$. 

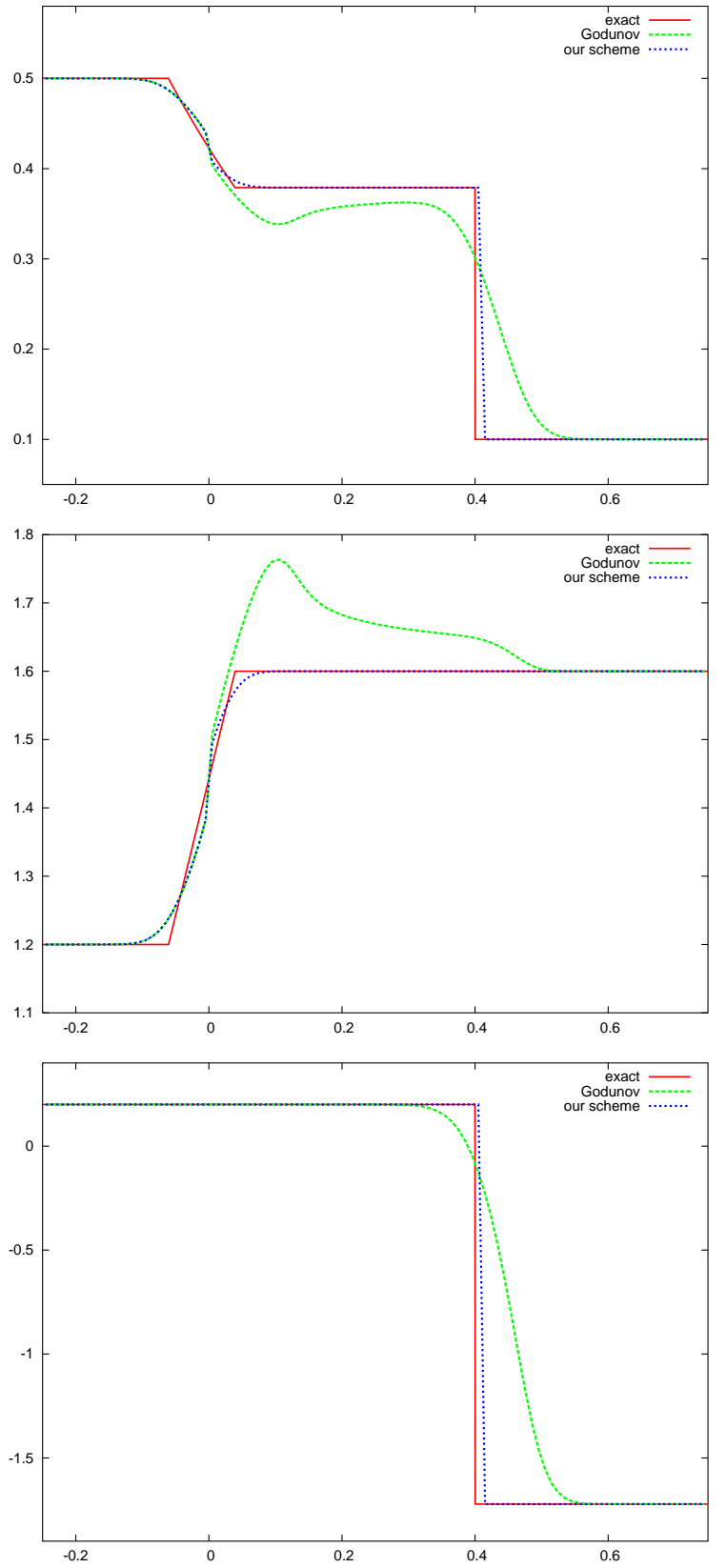

Fig. 5.3. Test 3: $\rho$ (Left), $v$ (Right) and $v+p(\rho)$ (Bottom) at time $t=0.25$. 


\begin{tabular}{ccccc}
\hline \# of points & $E_{\text {cons }}^{\rho}$ & $E_{\text {cons }}^{y}$ & $E_{L^{1}}^{\rho}$ & $E_{L^{1}}^{v}$ \\
\hline 100 & 0. & 0. & $8.39 e^{-2}$ & $8.68 e^{-2}$ \\
\hline 500 & 0. & 0. & $4.26 e^{-2}$ & $3.83 e^{-2}$ \\
\hline 1000 & 0. & 0. & $3.06 e^{-2}$ & $2.66 e^{-2}$ \\
\hline 2000 & 0. & 0. & $2.18 e^{-2}$ & $1.85 e^{-2}$ \\
\hline \# of points & $E_{\text {cons }}^{\rho}$ & $E_{\text {cons }}^{y}$ & $E_{L^{1}}^{\rho}$ & $E_{L^{1}}^{v}$ \\
\hline 100 & $1.52 \%$ & $7.74 \%$ & $8 . e^{-3}$ & 0. \\
\hline 500 & $0.32 \%$ & $1.83 \%$ & $1.6 e^{-3}$ & 0. \\
\hline 1000 & $0.16 \%$ & $0.94 \%$ & $8 . e^{-4}$ & 0. \\
\hline 2000 & $0.08 \%$ & $0.47 \%$ & $4 . e^{-4}$ & 0.
\end{tabular}

TABle 5.1. Test 1: Godunov scheme (top) and our scheme (bottom).

\begin{tabular}{ccccc}
\hline \# of points & $E_{\text {cons }}^{\rho}$ & $E_{\text {cons }}^{y}$ & $E_{L^{1}}^{\rho}$ & $E_{L^{1}}^{v}$ \\
\hline 100 & 0. & 0. & $3.2 e^{-3}$ & $6.55 e^{-3}$ \\
\hline 500 & 0. & 0. & $1.47 e^{-3}$ & $2.76 e^{-3}$ \\
\hline 1000 & 0. & 0. & $1.03 e^{-3}$ & $1.78 e^{-3}$ \\
\hline 2000 & 0. & 0. & $7.3 e^{-4}$ & $1.22 e^{-3}$ \\
\hline \hline \# of points & $E_{\text {cons }}^{\rho}$ & $E_{\text {cons }}^{y}$ & $E_{L^{1}}^{\rho}$ & $E_{L^{1}}^{v}$ \\
\hline 100 & $0.35 \%$ & $0.14 \%$ & $1.02 e^{-3}$ & $2.3 e^{-3}$ \\
\hline 500 & $0.07 \%$ & $0.03 \%$ & $2.19 e^{-4}$ & $6.47 e^{-4}$ \\
\hline 1000 & $0.04 \%$ & $0.02 \%$ & $1.09 e^{-4}$ & $3.26 e^{-4}$ \\
\hline 2000 & $0.03 \%$ & $0.01 \%$ & $9.72 e^{-5}$ & $1.63 e^{-4}$ \\
\hline
\end{tabular}

TABle 5.2. Test 2: Godunov scheme (top) and our scheme (bottom).

\begin{tabular}{ccccc}
\hline \# of points & $E_{\text {cons }}^{\rho}$ & $E_{\text {cons }}^{y}$ & $E_{L^{1}}^{\rho}$ & $E_{L^{1}}^{v}$ \\
\hline 100 & 0. & 0. & $2.12 e^{-2}$ & $3.8 e^{-2}$ \\
\hline 500 & 0. & 0. & $9.82 e^{-3}$ & $1.68 e^{-2}$ \\
\hline 1000 & 0. & 0. & $6.98 e^{-3}$ & $1.17 e^{-2}$ \\
\hline 2000 & 0. & 0. & $4.94 e^{-3}$ & $8.22 e^{-3}$ \\
\hline \# of points & $E_{\text {cons }}^{\rho}$ & $E_{\text {cons }}^{y}$ & $E_{L^{1}}^{\rho}$ & $E_{L^{1}}^{v}$ \\
\hline 100 & $0.81 \%$ & $6.04 \%$ & $3.82 e^{-3}$ & $3.36 e^{-3}$ \\
\hline 500 & $0.17 \%$ & $1.14 \%$ & $9.41 e^{-4}$ & $1.25 e^{-3}$ \\
\hline 1000 & $0.08 \%$ & $0.57 \%$ & $5.17 e^{-4}$ & $7.78 e^{-4}$ \\
\hline 2000 & $0.04 \%$ & $0.28 \%$ & $2.84 e^{-4}$ & $4.72 e^{-4}$ \\
\hline
\end{tabular}

TABle 5.3. Test 3: Godunov scheme (top) and our scheme (bottom). 


\section{REFERENCES}

[1] R. Abgrall, How to prevent pressure oscillations in multicomponent flow calculations: a quasiconservative approach, J. Comput. Phys., 125, 150-160, 1996.

[2] R. Abgrall and S. Karni, Computations of compressible multifluids, J. Comput. Phys., 169, 594-623, 2001.

[3] A. Aw and M. Rascle, Resurrection of "second order" models of traffic flow, SIAM J. Appl. Math., 60, 916-938, 2000.

[4] T. Barberon, P. Helluy and S. Rouy, Practical computation of axisymmetrical multifluid flows, International Journal of Finite Volumes, 1, 1-34, 2003.

[5] C. Chalons, Transport-equilibrium schemes for computing nonclassical shocks. scalar conservation laws, preprint, available at http://www.math.ntnu.no/conservation/2006/051.html, 2006.

[6] C. Chalons, Numerical approximation of a macroscopic model of pedestrian flows, SIAM J. Sci. Comput., 2007.

[7] C. Chalons and F. Coquel, Numerical contact discontinuities in single fluid and multifluid computations, preprint.

[8] C. Chalons and P. Goatin, Godunov scheme and sampling technique for computing phase transitions in traffic flow modeling, preprint, available at http://www.math.ntnu.no/ conservation/2006/052.html, 2006.

[9] P. Collela, Glimm's method for gas dynamics, SIAM J. Sci. Stat. Comput., 3, 76-110, 1982.

[10] R.M. Colombo, A $2 \times 2$ hyperbolic traffic flow model, Math. Comput. Modeling, 35(5-6), 683688,2002

[11] R.M. Colombo, Hyperbolic phase transitions in traffic flow, SIAM J. Appl. Math., 63(2), 708$721,2002$.

[12] C.F. Daganzo, Requiem for high-order fluid approximations of traffic flow, Trans. Res., 29B(4), 277-287, 1995.

[13] R.P. Fedkiw, T. Aslam, B. Merriman and S. Osher, A non-oscillatory eulerian approach to interfaces in multimaterial flows (the ghost fluid method), J. Comput. Phys., 152, 457-492, 1999.

[14] P. Goatin, The Aw-Rascle traffic flow model with phase transition, Math. Comput. Modeling, 44, 287-303, 2006.

[15] S. Karni, Multicomponent flow calculations by a consistent primitive algorithm, J. Comput. Phys., 47, 1115-1145, 1994.

[16] S. Karni, Hybrid multifluid algorithms, SIAM J. Sci. Comput., 17, 1019-1039, 1996.

[17] M.J. Lighthill and G.B. Whitham, On kinematic waves. II. a theory of traffic flow on long crowded roads, Proc. Roy. Soc. London. Ser. A., 229, 317-345, 1955.

[18] T.P. Liu, The deterministic version of the Glimm scheme, Comm. Math. Phys., 57, 135-148, 1977.

[19] H.J. Payne, Models of freeway traffic and control, in Simulation Council Proc. 28, Math. Models Publ. Sys., 51-61, 1971.

[20] P.I. Richards, Shock waves on the highway, Operations Res., 4, 42-51, 1956.

[21] R. Saurel and R. Abgrall, A simple method for compressible multifluid flows, SIAM J. Sci. Comput., 21, 1115-1145, 1999.

[22] D. Serre, Systèmes de lois de conservation, Diderot éditeur, Arts et Sciences, 304, 1996.

[23] B. Temple, Systems of conservation laws with coinciding shock and rarefaction curves, Contemp. Math., 17, 143-151, 1983.

[24] G.B. Whitham, Linear and Nonlinear Waves, John Wiley and Sons, New York, 1999. 